\title{
Evolução Temporal do Perfil de Risco e Resultados da Intervenção Coronariana Percutânea em Pacientes com Disfunção Ventricular Esquerda: Dados da Central Nacional de Intervenções Cardiovasculares (CENIC)
}

\author{
Cristiano de Oliveira Cardoso ${ }^{1,2}$, Alexandre Schaan de Quadros ${ }^{1,2}$, Rogério Eduardo Gomes Sarmento-Leite ${ }^{1,2}$, \\ Carlos Antônio Mascia Gottschall1,2, Luiz Alberto Mattos ${ }^{2,3}$, José Antonio Marin-Neto ${ }^{2,4}$
}

\section{RESUMO}

Introdução: Estudos antigos estabeleceram que pacientes com risco elevado seriam beneficiados quando tratados por cirurgia de revascularização miocárdica. Entretanto, não é tão claro se esse benefício também poderia ser alcançado por intervenções coronarianas percutâneas (ICP). O objetivo do presente estudo foi avaliar os resultados imediatos das ICP realizadas em pacientes com disfunção ventricular esquerda e o perfil geral de risco dos pacientes assim tratados. Método: Com base no registro Central Nacional de Intervenções Cardiovasculares (CENIC), foram analisadas todas as ICP realizadas em pacientes com disfunção ventricular esquerda entre 1999 e 2007. Os pacientes foram divididos em dois grupos: grupo A (antes de 2002) e grupo B (depois de 2002). Características clínicas, angiográficas e desfechos da fase hospitalar foram comparados. Análise estatística comparando os dois grupos foi realizada com teste de qui-quadrado e teste $t$ de Student, sendo significativo $P<0,05$. Resultados: Foram analisados 50.587 pacientes tratados por ICP $(n=12.783$ no grupo A e $\mathrm{n}=37.804$ no grupo B). Registrou-se perfil geral de risco significativamente mais elevado no grupo $\mathrm{B}$, bem como maior número de intervenções em lesões complexas e em pacientes com disfunção ventricular esquerda moderada e grave. Ocorreu redução dos índices de óbito hospitalar $(2,4 \%$ vs. $1,6 \% ; \mathrm{P}<0,001)$, infarto agudo do miocárdio $(0,9 \%$ vs. $0,5 \% ; \mathrm{P}<0,001)$, acidente vascular encefálico $(2,2 \%$ vs. $0,4 \% ; P<0,001)$, insuficiência renal aguda $(2,7 \%$ vs. $0,8 \% ; \mathrm{P}<0,001)$ e complicações vasculares $(6,3 \%$ vs. $1,6 \% ; P<0,001)$. Conclusão: Este estudo, focando exclusivamente pacientes com

\section{ABSTRACT}

Temporal Evolution of Risk Profile and Outcomes of Percutaneous Coronary Intervention in Patients with Left Ventricular Dysfunction: Results from the National Registry of Cardiovascular Interventions (CENIC)

Background: Previous authors demonstrated better outcomes for patients with left ventricular dysfunction (LVD) undergoing coronary artery bypass grafting. However, it is not yet clear if those patients could benefit from percutaneous coronary intervention ( $\mathrm{PCl})$. This study aimed to evaluate the early $\mathrm{PCl}$ outcomes in patients with LVD undergoing $\mathrm{PCl}$. Methods: The database of the National Registry of Cardiovascular Interventions (CENIC) of the Brazilian Society of Hemodynamics and Interventional Cardiology (SBHCl) was used to analyze all $\mathrm{PCl}$ procedures in patients with LVD between 1999-2007. Patients were divided in two groups: group A (before 2002) and group B (after 2002). Clinical and angiographic characteristics and in-hospital outcomes were recorded and compared. Statistical analyses were performed using the chi-square test and Student's $t$ test, and $\mathrm{P}<0.05$ was considered statistically significant. Results: A total of 50,587 patients were treated by $\mathrm{PCl}$ ( $\mathrm{n}=12,783$ in Group $\mathrm{A}$ and $\mathrm{n}=37,804$ in Group B). There was a significantly higher risk profile in group $\mathrm{B}$, as well as a higher number of $\mathrm{PCl}$ in complex lesions and in patients with moderate and severe LVD. Rates of in-hospital mortality $(2.4 \%$ vs. $1.6 \% ; \mathrm{P}<0.001)$, acute myocardial infarction $(0.9 \%$ vs. $0.5 \% ; \mathrm{P}<0.001)$ and stroke $(2.2 \% \times 0.4 \% ; \mathrm{P}<0.001)$ were reduced in patients

Instituto de Cardiologia do Rio Grande do Sul - Porto Alegre, RS, Brasil.

Central Nacional de Intervenções Cardiovasculares (CENIC) - São Paulo, SP, Brasil.

3 Instituto Dante Pazzanese de Cardiologia - São Paulo, SP, Brasil.

${ }^{4}$ Hospital das Clínicas da Faculdade de Medicina de Ribeirão Preto (USP) - Ribeirão Preto, SP, Brasil.

Correspondência: Cristiano de Oliveira Cardoso. Rua Anita Garibaldi, 2.381/707 - Boa Vista - Porto Alegre, RS, Brasil - CEP 90480-201

E-mail: co.cardoso@terra.com.br

Recebido em: 30/7/2008 • Aceito em: 16/2/2009 
Cardoso CO, et al. Evolução Temporal do Perfil de Risco e Resultados da Intervenção Coronariana Percutânea em Pacientes com Disfunção Ventricular Esquerda: Dados da Central Nacional de Intervenções Cardiovasculares (CENIC). Rev Bras Cardiol Invas. $2009 ; 17(1): 58-68$.

disfunção ventricular esquerda, mostra que resultados adequados das ICP em hospital foram obtidos e mantidos a despeito da progressiva elevação do perfil geral de risco nos pacientes assim tratados, ao longo do período de 1999 a 2007.

DESCRITORES: Angioplastia transluminal percutânea coronária. Stents. Fatores de risco. Função ventricular. Disfunção ventricular esquerda/terapia. treated after 2002. Conclusion: This study shows that adequate $\mathrm{PCl}$ results were obtained and maintained in hospitalized patients with LVD despite the progressive increase in the overall risk profile of these patients from 1999 to 2007.

DESCRIPTORS: Angioplasty, transluminal, percutaneous coronary. Stents. Risk factors. Ventricular function. Ventricular dysfunction, left/therapy.
$\mathrm{H}$ istoricamente, primeiro se estabeleceu o conceito de que pacientes com lesões graves em tronco de artéria coronária esquerda, disfunção ventricular esquerda, diabetes melito e lesões multivasculares se beneficiariam de cirurgia de revascularização do miocárdio, em comparação com os tratados clinicamente (com os recursos da época correspondente) ${ }^{1}$. Posteriormente, durante os primórdios da intervenção coronariana percutânea (ICP), fundamentalmente realizada com balões, sem uso de stents, estudos em grupos de pacientes com aquelas características sugeriram superioridade da cirurgia de revascularização do miocárdio sobre a ICP ${ }^{2-7}$. No entanto, observa-se aumento crescente dos procedimentos percutâneos em pacientes com disfunção ventricular esquerda, e pacientes anteriormente encaminhados à cirurgia de revascularização do miocárdio hoje são tratados percutaneamente $^{8-10}$.

Além de o arsenal técnico usado durante a ICP ser muito pouco desenvolvido na época daqueles estudos, o tratamento clínico adjunto também evoluiu bastante a partir da década de 1990. A combinação dessa evolução com o desenvolvimento técnico constante na área da cardiologia intervencionista repercutiu em aumento dos índices de sucesso e redução das taxas de complicações da ICP. Em especial, o advento dos stents farmacológicos trouxe novo alento para o tratamento por meio de ICP de pacientes antes encaminhados a cirurgia de revascularização do miocárdio ${ }^{11-13}$.

Embora se careça de dados sobre procedimentos no Brasil, na literatura internacional publicações tanto mais precoces como mais recentes testemunham os bons resultados alcançados com ICP em pacientes com disfunção ventricular esquerda ${ }^{14,15}$. O objetivo do presente estudo, focalizando essencialmente pacientes com disfunção ventricular esquerda submetidos a ICP, foi determinar o comportamento temporal do perfil geral de risco nesse grupo de doentes, e o correspondente conjunto de resultados obtidos na fase hospitalar dos procedimentos, conforme dados da Central Nacional de Intervenções Cardiovasculares (CENIC).

\section{MÉTODO \\ O Registro CENIC}

Os dados relacionados aos procedimentos no presente estudo foram obtidos a partir do banco de dados da CENIC, mantido pela Sociedade Brasileira de Hemodinâmica e Cardiologia Intervencionista ( $\mathrm{SBHCl}$ ). Os relatos individuais de procedimentos são voluntariamente enviados pelos membros da Sociedade e incluídos nesse registro. A SBHCl estimula seus sócios a responder e enviar um questionário com detalhes técnicos de todas as intervenções realizadas, coronarianas ou valvulares. O formulário do registro CENIC para ICP contém informações referentes a fatores de risco para cardiopatia isquêmica, características clínicas dos pacientes, detalhes técnicos do procedimento realizado, e evolução clínica dos pacientes na fase hospitalar. Todo o processo é atualmente enviado via Internet por meio de um formulário padrão e atualizado periodicamente.

\section{Seleção de pacientes}

Os dados relacionados aos procedimentos de ICP em pacientes com disfunção ventricular esquerda incluídos neste estudo foram obtidos do banco de dados CENIC entre os anos de 1999 e 2007. Para evitar qualquer viés de seleção, não foram aplicados critérios de exclusão e todas as intervenções cadastradas em pacientes com disfunção ventricular esquerda foram incluídas na análise. As análises foram feitas conforme o ano em que o procedimento foi realizado, dividindo-se os pacientes em dois grupos: grupo A, pacientes submetidos a ICP até 2001 (inclusive); e grupo B, pacientes submetidos a ICP no período de 2002 a 2007. Foi a partir de 2002 que os stents farmacológicos passaram a ser disponibilizados no Brasil, de forma que esse critério permitirá considerar o impacto desse fator nos resultados a serem analisados.

\section{Características analisadas}

De acordo com as informações registradas na CENIC, foram coletadas e analisadas informações contidas nas telefichas referentes a idade, gênero, fatores de risco 
Cardoso CO, et al. Evolução Temporal do Perfil de Risco e Resultados da Intervenção Coronariana Percutânea em Pacientes com Disfunção Ventricular Esquerda: Dados da Central Nacional de Intervenções Cardiovasculares (CENIC). Rev Bras Cardiol Invas. 2009;17(1):58-68.

para doença cardiovascular (diabetes melito, hipertensão arterial, tabagismo, dislipidemia, história familiar), apresentação clínica e indicação da ICP, categorização da disfunção ventricular (em leve, moderada e grave), número de vasos comprometidos, vaso-alvo da ICP, características angiográficas da lesão, e índices de sucesso e de complicações (óbito, complicações de acesso vascular, infarto agudo do miocárdio, insuficiência renal após ICP e acidente vascular encefálico).

\section{Análise estatística}

Os dados são apresentados em média, desvio padrão e porcentual. Para comparação das variáveis categóricas, foi utilizado o teste de qui-quadrado, e as variáveis contínuas foram analisadas pelo teste $t$ de Student, considerando nível de significância $P<0,05$.

\section{RESULTADOS}

Entre os anos de 1999 e 2007, foram reportados ao registro CENIC 50.587 procedimentos para tratamento de doença arterial coronariana em pacientes com disfunção ventricular esquerda. Foram registradas 12.783 (grupo A) e 37.804 (grupo B) ICPs no primeiro e segundo períodos estudados, respectivamente.

Com relação ao perfil dos pacientes, observou-se padrão heterogêneo de complexidade na comparação dos dois grupos, considerando-se as características estudadas. Assim, o perfil de risco clínico global dos doentes apresentou significativo aumento de gravidade no grupo B, comparativamente ao grupo A: apenas as taxas de hipertensão arterial foram comparáveis nos dois grupos, observando-se para o grupo B incrementos significativos na prevalência de tabagismo, dislipidemia, diabetes melito, antecedentes de infarto do miocárdio e história familiar positiva para cardiopatia isquêmica. Notou-se também no grupo B aumento da prevalência de pacientes que sofreram procedimentos prévios de revascularização, seja por cirurgia ou angioplastia. Detectou-se ainda, no grupo B, que a ICP foi mais frequentemente realizada em pacientes com síndrome isquêmica aguda de médio e alto riscos, quando aplicados os critérios do escore de risco TIMI. Essas características clínicas dos pacientes submetidos a ICP são apresentadas na Tabela 1.

Na Tabela 2, são mostradas as características angiográficas dos procedimentos. No total, foram tratadas 17.118 e 53.144 lesões nos períodos compreendidos antes e depois de 2001, respectivamente. Em comparação ao grupo A, observou-se significativo aumento do número de lesões tratadas por paciente (1,3 lesão por paciente no grupo A vs. 1,4 lesão por paciente no grupo $\mathrm{B} ; \mathrm{P}=0,002)$. O número de angioplastias com implante de stents também foi significativamente maior a partir de 2002 (73,5\% vs. 87,24\%; $\mathrm{P}<0,001)$. E foi também a partir de 2002 que se verificou a incorporação dos stents farmacológicos na prática clínica, evidenciada pelo uso crescente dessa tecnologia $(0,72 \%$ no grupo A vs. $12,7 \%$ no grupo $B$; $\mathrm{P}<0,001)$.

Acompanhando a mesma tendência do perfil clínico de risco mais grave no grupo $\mathrm{B}$, o padrão angiográfico também apresentou diferenças significantes entre os dois períodos. Lesões complexas do tipo C, lesões com comprimento $>20 \mathrm{~mm}$ e envolvimento de ramos laterais foram encontrados com maior frequência no grupo B. O tronco de artéria coronária esquerda também foi significativamente mais abordado no grupo B, bem como pacientes apresentando comprometimento multivascular, que foram tratados em maior proporção no período de 2002 em diante. As características angiográficas são detalhadamente mostradas na Tabela 2.

Com relação aos desfechos, os dados revelam equiparação nas taxas de sucesso imediato do procedimento. Fato estatisticamente significante foi a redução das complicações no grupo B. Observou-se expressiva queda da mortalidade imediata, bem como queda das taxas de infarto relacionado, insuficiência renal, complicações vasculares e acidente vascular encefálico na fase hospitalar após o procedimento. Na Tabela 3, são descritos os desfechos dos procedimentos nos dois períodos assinalados.

\section{DISCUSSÃO}

Publicações anteriores, divulgando resultados das ICPs no plano nacional, têm sido oriundas do registro CENIC ${ }^{16-21}$. Tanto dados de procedimentos eletivos quanto casos de infarto agudo do miocárdio foram anteriormente reportados, fato importante para a divulgação do que tem sido realizado pelos membros da $\mathrm{SBHCI}$. Entretanto, não havia relatos sobre o estado atual das ICPs em pacientes com disfunção ventricular esquerda.

Os pacientes com disfunção ventricular esquerda fazem parte de crescente subgrupo específico na prática cardiológica atual. Por apresentarem mortalidade aumentada, em diversos cenários clínicos e intervencionistas, se comparados a pacientes com fração de ejeção normal ${ }^{15}$, os doentes com essa característica são, geralmente, excluídos da maioria dos ensaios clínicos randomizados. Além disso, o benefício da revascularização está intimamente relacionado com a presença ou não de viabilidade miocárdica ${ }^{22,23}$. Carece-se, portanto, de evidências robustas nesse subgrupo de indivíduos.

Historicamente, registra-se o conceito de que pacientes com menor fração de ejeção seriam mais beneficiados por cirurgia de revascularização do miocárdio, em cotejo com revascularização por via percutânea. Entretanto, foram dados da década de $90^{2-7,24}$ que demonstravam resultados favoráveis aos pacientes tratados por cirurgia quando comparados aos submetidos a angioplastia. É importante salientar que esses estudos demonstravam essencialmente a superioridade do procedimento cirúrgico no que tange à sobrevida livre 
Cardoso CO, et al. Evolução Temporal do Perfil de Risco e Resultados da Intervenção Coronariana Percutânea em Pacientes com Disfunção Ventricular Esquerda: Dados da Central Nacional de Intervenções Cardiovasculares (CENIC). Rev Bras Cardiol Invas. 2009;17(1):58-68.

TABELA 1

Características clínicas dos pacientes com disfunção ventricular esquerda submetidos a intervenção coronariana percutânea

\begin{tabular}{|c|c|c|c|}
\hline & $\begin{array}{c}\text { Grupo A } \\
\text { Antes de } 2002(1999-2001) \\
n=12.783\end{array}$ & $\begin{array}{c}\text { Grupo B } \\
\text { Depois de } 2002(2002-2007) \\
n=37.804\end{array}$ & $\mathbf{P}$ \\
\hline \multicolumn{4}{|l|}{ Características clínicas (\%) } \\
\hline Homens & 70,4 & 70,1 & 0,7 \\
\hline Diabetes melito & 15,7 & 32,2 & $<0,001$ \\
\hline Hipertensão arterial & 77,0 & 77,9 & 0,9 \\
\hline Tabagismo & 23,0 & 30,7 & $<0,001$ \\
\hline Dislipidemia & 22,6 & 61 & $<0,001$ \\
\hline História familiar de DAC & 23,1 & 32,1 & $<0,001$ \\
\hline IAM prévio & 21,0 & 38,3 & $<0,001$ \\
\hline ICP prévia & 19,7 & 27,1 & $<0,001$ \\
\hline CRM prévia & 19,8 & 22,1 & $<0,001$ \\
\hline Apresentação clínica (\%) & & & $<0,001$ \\
\hline Assintomático & 4,8 & 5,4 & \\
\hline Angina estável & 27,4 & 32,9 & \\
\hline SCA sem supra ST & 46,1 & 36,9 & \\
\hline IAM com supra ST & 21,7 & 24,5 & \\
\hline SCA sem supra ST & & & $<0,001$ \\
\hline Risco baixo & 67,9 & 25,9 & \\
\hline Risco médio & 24,7 & 46,8 & \\
\hline Risco alto & 7,4 & 27,1 & \\
\hline IAM com supra ST & & & $<0,001$ \\
\hline Killip I-II & 76,2 & 87,4 & \\
\hline Killip III-IV & 23,8 & 12,6 & \\
\hline Disfunção da FV & & & $<0,01$ \\
\hline Leve & 56,5 & 55,3 & \\
\hline Moderada & 32,5 & 32,9 & \\
\hline Grave & 10,9 & 11,7 & \\
\hline
\end{tabular}

CRM = cirurgia de revascularização miocárdica; $D A C=$ doença arterial coronariana; $F V=$ função ventricular; IAM = infarto agudo do miocárdio; ICP = intervenção coronariana percutânea; $\mathrm{n}=$ número de pacientes; SCA = síndrome coronariana aguda.

de angina; em adição, por meio de subanálises, sugeria-se também benefício em termos de mortalidade da cirurgia de revascularização do miocárdio sobre a ICP para tratar pacientes com disfunção ventricular esquerda. Entretanto, tais estudos refletem fundamentalmente os resultados obtidos na era da angioplastia com balão. Em contraste com esse panorama inicial das comparações, estudos mais recentes ${ }^{8,14,15}$ têm demonstrado benefício cada vez mais nítido da ICP realizada em pacientes com disfunção ventricular esquerda. É importante assinalar que nesse grupo de pacientes com disfunção ventricular esquerda, mesmo sem se elevar significativamente a fração de ejeção, a ICP costuma promover expressiva melhora no remodelamento ventricular, por meio de redução dos volumes telediastólico e telessistólico ${ }^{25}$. Esses resultados tendem a amplificar os benefícios da revascularização por via percutânea realizados em pacientes com disfunção ventricular esquerda, e a credenciam a obter resultados comparáveis aos obtidos por meio de cirurgia de revascularização do miocárdio. Ainda mais recentemente, com a introdução dos stents farmacológicos no cenário das intervenções, observa-se que essa tecnologia tem se mostrado segura e eficaz em pacientes com fração de ejeção reduzida ${ }^{9,10}$. Além disso, no Brasil, o uso dos stents farmacológicos tem crescido progressivamente, conforme publicação recente de resultados da CENIC ${ }^{21}$. Nesse estudo, o nítido incremento anual no uso desse dispositivo para ICP espelhou exatamente o que vem acontecendo no cenário mundial nos últimos anos. Esses dados estão em consonância com os resultados do registro internacional Can Rapid Risk Stratification of Unstable Angina Patients Suppress Adverse Outcomes With Early Implementation 
Cardoso CO, et al. Evolução Temporal do Perfil de Risco e Resultados da Intervenção Coronariana Percutânea em Pacientes com Disfunção Ventricular Esquerda: Dados da Central Nacional de Intervenções Cardiovasculares (CENIC). Rev Bras Cardiol Invas. 2009;17(1):58-68.

TABELA 2

Características angiográficas dos pacientes com disfunção ventricular esquerda submetidos a intervenção percutânea

\begin{tabular}{|c|c|c|c|}
\hline & $\begin{array}{c}\text { Grupo A } \\
\text { Antes de } 2002(1999-2001) \\
n=12.783\end{array}$ & $\begin{array}{c}\text { Grupo B } \\
\text { Depois de } 2002(2002-2007) \\
n=37.804\end{array}$ & $\mathbf{P}$ \\
\hline Número de vasos afetados (\%) & & & $<0,001$ \\
\hline Até dois & 62,4 & 59,6 & \\
\hline Três & 37,4 & 40,1 & \\
\hline TCE & 0,007 & 0,15 & \\
\hline Vasos tratados $(\%)$ & & & 0,08 \\
\hline DA & 31 & 32,1 & \\
\hline $\mathrm{CX}$ & 15,7 & 16 & \\
\hline$C D$ & 29,1 & 29 & \\
\hline TCE & 1 & 1,2 & \\
\hline Enxerto venoso e arterial & 6,3 & 5,7 & \\
\hline Outros & 16,4 & 15,8 & \\
\hline Padrão da lesão (\%) & & & $<0,001$ \\
\hline A & 3,2 & 3,7 & \\
\hline B1 & 27 & 27 & \\
\hline B2 & 46,6 & 43,1 & \\
\hline $\mathrm{C}$ & 23 & 26 & \\
\hline Comprimento > $20 \mathrm{~mm}$ & 20 & 31,3 & $<0,001$ \\
\hline Ramos envolvidos & 28,6 & 29,2 & 0,2 \\
\hline \multicolumn{4}{|l|}{ Angioplastia (\%) } \\
\hline Com stent & 73,5 & 87,4 & $<0,001$ \\
\hline Só balão & 28,6 & 12,5 & $<0,001$ \\
\hline
\end{tabular}

A, B1, B2, C = padrão de lesão, de acordo com a classificação da AHA/ACC; CD = artéria coronária direita; CX = artéria circunflexa; $\mathrm{DA}=$ artéria descendente anterior; $\mathrm{n}=$ número de pacientes; $\mathrm{TCE}=$ tronco de artéria coronária esquerda.

of the ACC/AHA (American College of Cardiology/American Heart Association) Guidelines (CRUSADE) ${ }^{26}$, demonstrando que o número de ICPs cresceu significativamente e reduziu os índices de tratamento clínico e revascularização cirúrgica nos pacientes com lesões triarteriais, após a aprovação dos stents farmacológicos nos Estados Unidos.

Com o presente estudo, abordando-se especificamente pacientes que apresentavam algum grau de disfunção ventricular esquerda, o que mais chama a atenção é o expressivo agravamento recente no perfil geral de risco dos pacientes com disfunção ventricular esquerda que são tratados por ICP. Em comparação com o ocorrido até 2001, a prevalência de fatores de risco clássicos aumentou em geral a partir de 2002, tornando o perfil de risco dos pacientes de maior gravidade. Observou-se, assim, significativo aumento da prevalência de tabagismo, dislipidemia, diabetes melito e também infarto agudo do miocárdio, ICP e cirurgia de revascularização do miocárdio prévias. Quando avaliados pelo perfil de risco $\mathrm{TIMI}^{27}$, ocorreu progressivo aumento numérico de pacientes com angina instável de médio e alto riscos. O padrão angiográfico também apresentou características de maior gravidade, sendo a intervenção de tronco de artéria coronária esquerda realizada mais comumente a partir de 2002.

Apesar desse cenário clínico e angiográfico de mais elevado risco no perfil dos pacientes e das condições técnicas dos procedimentos, os resultados imediatos da ICP foram mantidos em níveis elevados e comparáveis nos dois períodos estudados neste trabalho com os dados da CENIC. Isso ocorreu não obstante o fato de que, em geral, a mortalidade intra-hospitalar em pacientes submetidos a ICP previsivelmente tende a ser proporcionalmente mais elevada e pode ser realmente estimada com base em características clínicas e angiográficas de maior risco $^{28}$. Dessa forma, outros fatores de evolução técnica dos procedimentos devem ter contrabalançado a tendência a maior mortalidade derivada do maior risco inerente, nesta amostra substancial de pacientes arrolados no registro CENIC. Além da mortalidade, também as outras complicações durante a fase hospitalar (complica- 
Cardoso CO, et al. Evolução Temporal do Perfil de Risco e Resultados da Intervenção Coronariana Percutânea em Pacientes com Disfunção Ventricular Esquerda: Dados da Central Nacional de Intervenções Cardiovasculares (CENIC). Rev Bras Cardiol Invas. 2009;17(1):58-68.

TABELA 3

Desfecho dos procedimentos em pacientes com disfunção ventricular esquerda submetidos a intervenção percutânea

\begin{tabular}{|c|c|c|c|}
\hline & $\begin{array}{c}\text { Grupo A } \\
\text { Antes de } 2002(1999-2001) \\
n=12.783\end{array}$ & $\begin{array}{c}\text { Grupo B } \\
\text { Depois de } 2002(2002-2007) \\
n=37.804\end{array}$ & $\mathbf{P}$ \\
\hline \multicolumn{4}{|l|}{ Desfecho (\%) } \\
\hline Sucesso angiográfico & 98,7 & 98,8 & 0,9 \\
\hline Óbito & 2,4 & 1,6 & $<0,001$ \\
\hline IAM & 0,9 & 0,5 & $<0,001$ \\
\hline Revascularização de urgência & 16 & 15,2 & 0,07 \\
\hline AVC & 2,2 & 0,4 & $<0,001$ \\
\hline IRA & 2,7 & 0,8 & $<0,001$ \\
\hline Complicação vascular & 6,3 & 1,6 & $<0,001$ \\
\hline
\end{tabular}

AVC = acidente vascular encefálico; IAM = infarto agudo do miocárdio; IRA = insuficiência renal aguda; $\mathrm{n}=$ número de pacientes.

ções associadas ao acesso vascular, infarto agudo do miocárdio não-fatal e acidente vascular encefálico) diminuíram a partir de 2002, resultados esses alentadores quanto à segurança progressivamente mais elevada das intervenções realizadas em território nacional. Tais indícios de proficiência no uso de tecnologias e recursos médicos em geral associados aos procedimentos são particularmente relevantes nesse contexto mais ominoso da ICP, que é realizada em pacientes portando graus avançados de disfunção ventricular esquerda. Indicam, em essência, que o benefício da ICP pode ser estendido a muitos desses pacientes, sem necessidade de recorrer a cirurgia de revascularização do miocárdio, em diversos contextos em que este último tipo de procedimento se torna inexequível ou indesejável por razões de ordem médica ou social.

\section{Limitações do estudo}

Algumas limitações devem ser consideradas para a plena compreensão da presente análise:

1) As informações recebidas pela CENIC são enviadas por membros da $\mathrm{SBHCl}$ espontaneamente, logo um grande número de procedimentos realizados no Brasil não é reportado. Assim, os aspectos analisados não podem ser simplesmente atribuídos à totalidade dos procedimentos coronarianos percutâneos realizados em nosso País. Todavia, o elevado número de procedimentos aqui analisados torna este estudo bastante representativo da realidade brasileira.

2) A falta de seguimento a longo prazo impede qualquer conclusão com respeito à evolução tardia dos pacientes com disfunção ventricular esquerda tratados percutaneamente, tornando imperativa a necessidade de se evoluir o registro CENIC no sentido de dotá-lo de seguimento dos pacientes além da fase hospitalar da ICP.

\section{CONCLUSÃo}

Em relação ao período anterior a 2002, o perfil de risco clínico e angiográfico dos pacientes com disfunção ventricular esquerda recebendo ICP, registrados na CENIC, agravou-se substancialmente nos anos subsequentes. Apesar disso, muito provavelmente porque os procedimentos percutâneos realizados nesse segundo período de acompanhamento deste estudo (a partir de 2002) se beneficiaram de inúmeros aperfeiçoamentos tecnológicos e também de maior experiência acumulada, os resultados imediatos da ICP tornaram-se até melhores que antes de 2002.

\section{AGRADECIMENTO}

A presente análise só foi possível pelo constante envio de dados à CENIC durante o período estudado. Agradecemos, portanto, aos sócios da $\mathrm{SBHCl}$ que para tal contribuíram, cujos nomes estão anexos no final deste manuscrito.

\section{CONFLITO DE INTERESSES}

Cristiano de Oliveira Cardoso recebeu auxílio para participação em congressos da Biotronik. Alexandre Schaan de Quadros recebeu auxílio para pesquisa das empresas Boston Scientific e Medtronic, além de auxílio para participação em congressos da B-Braun e Medtronic. Rogério Eduardo G. Sarmento-Leite recebeu apoio educacional e de pesquisa das empresas Abbot, Biotronik e Cordis; Bureau de Palestrantes das empresas Biotronik e B-Braun; prestou consultoria técnica para Biotronik e pertence ao Comitê Científico da Scitech. Luiz Alberto Mattos foi palestrante em eventos ou atividades patrocinadas pelas seguintes indústrias: Abbott, Boston, Cordis, Biotronik e Medtronic; além disso, participou de estudos clínicos ou experimentais subvencionados pelas seguintes indústrias: Cordis, Biotronik, Medtronic e Biosensors. Os demais autores 
Cardoso CO, et al. Evolução Temporal do Perfil de Risco e Resultados da Intervenção Coronariana Percutânea em Pacientes com Disfunção Ventricular Esquerda: Dados da Central Nacional de Intervenções Cardiovasculares (CENIC). Rev Bras Cardiol Invas. 2009;17(1):58-68.

declararam inexistência de conflito de interesses com o presente estudo.

\section{REFERÊNCIAS BIBLIOGRÁFICAS}

1. Davis KB, Chaitman B, Ryan T, Bittner V, Kennedy JW. Comparison of 15-year survival for men and women after initial medical or surgical treatment for coronary artery disease: a CASS registry study. Coronary Artery Surgery Study. J Am Coll Cardiol. 1995;25(5):1000-9.

2. Coronary angioplasty versus coronary artery bypass surgery: the Randomized Intervention Treatment of Angina (RITA) trial. Lancet. 1993;341(8845):573-80.

3. CABRI Trial Participants. First-year results of CABRI (Coronary Angioplasty versus Bypass Revascularisation Investigation). Lancet. 1995;346(8984):1179-84.

4. The Bypass Angioplasty Revascularization Investigation (BARI) Investigators. Comparison of coronary bypass surgery with angioplasty in patients with multivessel disease. N Engl J Med. 1996;335(4):217-25.

5. Hamm CW, Reimers J, Ischinger T, Rupprecht HJ, Berger J, Bleifeld W. A randomized study of coronary angioplasty compared with bypass surgery in patients with symptomatic multivessel coronary disease. German Angioplasty Bypass Surgery Investigation (GABI). N Engl J Med. 1994;331(16):1037-43.

6. Henderson RA, Pocock SJ, Sharp SJ, Nanchahal K, Sculpher MJ, Buxton MJ, et al. Long-term results of RITA-1 trial: clinical and cost comparisons of coronary angioplasty and coronary-artery bypass grafting. Randomised Intervention Treatment of Angina. Lancet. 1998;352(9138):1419-25.

7. Rodriguez A, Boullon F, Perez-Baliño N, Paviotti C, Liprandi MI, Palacios IF. Argentine randomized trial of percutaneous transluminal coronary angioplasty versus coronary artery bypass surgery in multivessel disease (ERACI): in-hospital results and 1-year follow-up. ERACI Group. J Am Coll Cardiol. 1993;22(4):1060-7.

8. Caines AE, Massad MG, Kpodonu J, Rebeiz AG, Evans A, Geha AS. Outcomes of coronary artery bypass grafting versus percutaneous coronary intervention and medical therapy for multivessel disease with and without left ventricular dysfunction. Cardiology. 2004;101(1-3):21-8.

9. Gioia G, Matthai W, Benassi A, Rana H, Levite HA, Ewing LG. Improved survival with drug-eluting stent implantation in comparison with bare metal stent in patients with severe left ventricular dysfunction. Catheter Cardiovasc Interv. 2006; 68(3):392-8.

10. Gioia G, Matthai W, Gillin K, Dralle J, Benassi A, Gioia $M F$, et al. Revascularization in severe left ventricular dysfunction: outcome comparison of drug-eluting stent implantation versus coronary artery by-pass grafting. Catheter Cardiovasc Interv. 2007;70(1):26-33.

11. Dibra A, Kastrati A, Mehilli J, Pache J, Schuhlen H, von Beckerath $\mathrm{N}$, et al. Paclitaxel-eluting or sirolimus-eluting stents to prevent restenosis in diabetic patients. N Engl J Med. 2005;353(7):663-70.

12. Dibra A, Kastrati A, Alfonso F, Seyfarth M, Pérez-Vizcayno MJ, Mehilli J, et al. Effectiveness of drug-eluting stents in patients with bare-metal in-stent restenosis: meta-analysis of randomized trials. J Am Coll Cardiol. 2007;49(5):616-23.

13. Eisenberg MJ, Konnyu KJ. Review of randomized clinical trials of drug-eluting stents for the prevention of in-stent restenosis. Am J Cardiol. 2006;98(3):375-82.

14. Keelan PC, Johnston JM, Koru-Sengul T, Detre KM, Williams DO, Slater J, et al. Comparison of in-hospital and one-year outcomes in patients with left ventricular ejection fractions $<$ or $=40 \%, 41 \%$ to $49 \%$, and $>$ or $=50 \%$ having per- cutaneous coronary revascularization. Am J Cardiol. 2003; 91(10):1168-72.

15. Wallace TW, Berger JS, Wang A, Velazquez EJ, Brown DL. Impact of left ventricular dysfunction on hospital mortality among patients undergoing elective percutaneous coronary intervention. Am J Cardiol. 2009;103(3):355-60.

16. Sousa AG. Procedimentos Percutâneos de Intervenção Cardiovascular no Brasil em 1992 e 1993. Relatório do Registro Nacional - Central Nacional de Intervenções Cardiovasculares (CENIC). Arq Bras Cardiol. 1994;62(4):217-23.

17. Mattos LA, Sousa AG, Pinto IM, Silva ER, Carneiro JK, Sousa $J E$, et al. Uma comparação entre a intervenção coronariana percutânea de resgate e primária realizadas no infarto agudo do miocárdio. Relato multicêntrico de 9.371 pacientes. Arq Bras Cardiol. 2004;82(5):434-9.

18. Mattos LA, Sousa AG, Campos Neto CM, Labrunie A, Alves $C R$, Feres $F$, et al. The use of primary stenting or balloon percutaneous transluminal coronary angioplasty for the treatment of acutely occluded saphenous vein grafts. Results from the Brazilian National Registry-CENIC. Central Nacional de Intervenções Cardiovasculares. Arq Bras Cardiol. 2001;76(6): 483-95.

19. Mattos LA, Sousa AG, Neto CC, Labrunie A, Alves CR, Saad J. Primary coronary angioplasty and stent implantation in acute myocardial infarction. Comparative analysis of the inhospital results in the CENIC/SBHCl registry. National Center of Cardiovascular Interventions members. Arq Bras Cardiol. 1999;73(6):475-84.

20. Mangione J. Intervenção coronária percutânea no Brasil. Quais são os nossos números? Rev Bras Cardiol Invas. 2006; 14(3):267-72.

21. Cardoso CO, Quadros AS, Mattos LA, Gottschall CA, SarmentoLeite RE, Marin-Neto JA. Perfil de uso dos stents farmacológicos no Brasil: dados da Central Nacional de Intervenções Cardiovasculares (CENIC). Arq Bras Cardiol. 2007;89(6):356-61.

22. Allman KC, Shaw LJ, Hachamovitch R, Udelson JE. Myocardial viability testing and impact of revascularization on prognosis in patients with coronary artery disease and left ventricular dysfunction: a meta-analysis. J Am Coll Cardiol. 2002;39(7):1151-8.

23. Camici PG, Prasad SK, Rimoldi OE. Stunning, hibernation, and assessment of myocardial viability. Circulation. 2008; 117(1):103-14.

24. Pocock SJ, Henderson RA, Seed P, Treasure T, Hampton JR. Quality of life, employment status, and anginal symptoms after coronary angioplasty or bypass surgery. 3-year followup in the Randomized Intervention Treatment of Angina (RITA) Trial. Circulation. 1996;94(2):135-42.

25. Zellweger MJ, Tabacek G, Zutter AW, Weinbacher M, Cron TA, Muller-Brand J, et al. Evidence for left ventricular remodeling after percutaneous coronary intervention: effect of percutaneous coronary intervention on left ventricular ejection fraction and volumes. Int J Cardiol. 2004;96(2):197-201.

26. Gogo PB Jr., Dauerman HL, Mulgund J, Ohman EM, Patel MR, Cohen DJ, et al. Changes in patterns of coronary revascularization strategies for patients with acute coronary syndromes (from the CRUSADE Quality Improvement Initiative). Am J Cardiol. 2007;99(9):1222-6.

27. Antman EM, Cohen M, Bernink PJ, McCabe CH, Horacek T, Papuchis G, et al. The TIMI risk score for unstable angina/ non-ST elevation MI: a method for prognostication and therapeutic decision making. JAMA. 2000;284(7):835-42.

28. Wu C, Hannan EL, Walford G, Ambrose JA, Holmes DR Jr., King SB $3^{\text {rd }}$, et al. A risk score to predict in-hospital mortality for percutaneous coronary interventions. J Am Coll Cardiol. 2006;47(3):654-60. 


\section{APÊNDICE \\ Lista de Associados da SBHCI que se mantiveram efetivamente contribuindo com dados à CENIC durante o biênio 2006-2007}

ABDU NEME JORGE MAKHLUF NETO

ADEMAR SANTOS FILHO

ADNAN ALI SALMAN

ADRIANO DIAS DOURADO OLIVEIRA

ADRIANO MENDES CAIXETA

ALAN NASCIMENTO PAIVA

ALAOR QUEIROZ ARAUJO FILHO

ALBERTO NAJJAR

ALCIDES FERREIRA JUNIOR

ALCIDES JOSE ZAGO

ALDO FERNANDO SOMAVILLA DUARTE

ALESSANDRA OLIVEIRA

ALESSANDRO PINA PEDROSO

ALEXANDRE DA SILVA MEDEIROS

ALEXANDRE DAMIANI AZMUS

ALEXANDRE DO CANTO ZAGO

ALEXANDRE JACKSON VON SPERLING DE

VASCONCELLOS

ALEXANDRE LOJA ANELLO

ALEXANDRE SOARES DOS SANTOS

ALEXANDRE VENTURELLI

ALEXANDRE XAVIER BRANT

ALFREDO NUNES FERREIRA FILHO

ALUISIOCRUZJUNIOR

ALVARO DE MORAES JUNIOR

ALVARO LUIS MACHADO SOARES

ANA PAULA SCHER BARRETO LEAL

ANDERSON H. P. COSTA

ANDRE EDUARDO GOMES

ANDRE FRANCISCO DE PAULA ANTONANGELO

ANDRE GASPARINI SPADARO

ANDRE LABRUNIE

ANDRE LUIZ DA FONSECA FEIJO

ANDRE LUIZ SILVEIRA SOUSA

ANGELO AMATO VINCENZO DE PAOLA

ANSELMO ANTONIO SALGADO

ANTENOR LAGES FORTES PORTELA

ANTENORIO AIOLFI

ANTONIO CARLOS MANSUR BEDETI

ANTONIO CARLOS NEVES FERREIRA

ANTONIO CESAR DE SOUZA

ANTONIO DONIZETTI DE SENA PEREIRA

ANTONIO ESTEVES FILHO

ANTONIO FERNANDINO DE CASTRO BAHIA NETO

ANTONIO GILSON LAPA GODINHO

ANTONIO JOSE MUNIZ

ANTONIO MORAES DE AZEVEDO JUNIOR

ANTONIO WATSON MENESES VIANA

ARI MANDIL

ARTHUR LUIZ WALLBACH BARRETO

AUGUSTO DAIGE DA SILVA

AUGUSTO LIMA FILHO
BERNARDO AMORIM

BRENO DE ALENCAR ARARIPE FALCÃO

BRENO OLIVEIRA ALMEIDA

BRUNO MOULIN MACHADO

CARLAILE ANTONIO SARMENTO DE ARAUJO

COSTA

CARLOS AUGUSTO FORMIGA AREAS

CARLOS EDUARDO DINIZ COUTO

CARLOS EDUARDO FARIA SILVA

CARLOS EDUARDO MAGALHÃES DOMINGUES

CARLOS HENRIQUE EIRAS FALCÃO

CARLOS ROBERTO CARDOSO

CELMO FERREIRA DE SOUZA JUNIOR

CESAR AUGUSTO ESTEVES

CESAR ROCHA MEDEIROS

CHARLES LUIZ VIEIRA

CIRO JONES CARDOSO

CLACIR STAUDT

CLARISSA CAMPO DALL ORTO

CLAUDIA MARIA RODRIGUES ALVES

CLAUDIA MATTOS

CLAUDIO AKSTEIN

CLEITON DA SILVA RAMOS

CLEMENTE GREGUOLO

CONSTANTINO GONZALES SALGADO

COSTANTINO ROBERTO FRACK CONSTANTINI

CRISTIANO NONATO MADUREIRA LUCENA

CYRO VARGUES RODRIGUES

DANIEL FERREIRA MUGRABI

DANIEL IZZET POTERIO

DEBORAHCHRISTINA NERCOLINI

DECIOSALVADORI JUNIOR

DENIS MOULIN DOS REIS BAYERL

DIMITRI MIKAELIS ZAPPI

DINALDO CAVALCANTI DE OLIVEIRA

DIRCEU RODRIGUES ALMEIDA

DOMENICO RODRIGO GHINELLI ZAPATER

EDISON CARVALHO SANDOVAL PEIXOTO

EDMUNDO ANDRE VIVEIROS PESSANHA

EDMUR CARLOS DE ARAUJO

EDSON ADEMIR BOCCHI

EDSON ANTONIO BREGAGNOLLO

EDUARDO ARANTES NOGUEIRA

EDUARDO FREDERICO BORSARINI FELIPE

EDUARDO HENRIQUE CURADO ELIAS

EDUARDO JOSE PEREIRA FERREIRA

EDUARDO KEI MARQUESINI WASHIZU

EDUARDO LUCIO NICOLELA JUNIOR

EDUARDO SZUSTER

ELIAS DE MELLO AYRES NETO

EMERSON DE ALBUQUERQUE SEIXAS

ENIO EDUARDO GUERIOS 
Cardoso CO, et al. Evolução Temporal do Perfil de Risco e Resultados da Intervenção Coronariana Percutânea em Pacientes com Disfunção Ventricular Esquerda: Dados da Central Nacional de Intervenções Cardiovasculares (CENIC). Rev Bras Cardiol Invas. 2009;17(1):58-68.

\section{APÊNDICE \\ Lista de Associados da SBHCI que se mantiveram efetivamente contribuindo com dados à CENIC durante o biênio 2006-2007}

\begin{tabular}{|c|c|}
\hline EOLOMORANDI JUNIOR & GUSTAVO ANDRE BARRUECO \\
\hline ERIKA PRACCHIA RIBEIRO & GUSTAVO BAIOCHI VIEIRA \\
\hline ESMERALCI FERREIRA & GUSTAVO CARVALHO \\
\hline EULOGIO EMILIO MARTINEZ FILHO & GUSTAVO DE MORAES RAMALHO \\
\hline EUSDEMAR LIMA STEFANE & GUSTAVO ENRIQUE SANCHES ALVAREZ \\
\hline EVANDRO GOMES DE MATOS JUNIOR & GUSTAVO ITHAMAR SOUTO MAIOR \\
\hline EVANDRO KARLO PRACCHIA RIBEIRO & GUSTAVO OLIVEIRA DE ALBUQUERQUE \\
\hline EVANDRO LUIS QUEIROZ FLORES & GUSTAVO SOUTO MAIOR \\
\hline EXPEDITO EUSTAQUIO RIBEIRO DA SILVA & GYOVANY MENDES ALMEIDA \\
\hline FABIANO CARAZZAI PACHECO & HAROLDO CARLOS CORREA GLAVAM \\
\hline FABIO CARDOSO DE CARVALHO & HEITOR GHISSONI DE CARVALHO \\
\hline FABIO DE MEIRELES COSTA & HELIO JOSE CASTELLO JR. \\
\hline FABIO MONTEIRO MOTA & HELIO ROQUE FIGUEIRA \\
\hline FABIO RIDOLFI DE FIGUEIREDO & HELMAN CAMPOS MARTINS \\
\hline FÁBIO SÂNDOLI DE BRITO JR. & HENRIQUE ISSA ARTONI EBAID \\
\hline FABIO SOLANO DE FREITAS SOUZA & HUMBERTO ALENCAR DE ARAUJO SANCHEZ \\
\hline FAUSTO FERES & HUMBERTO MAGNO PASSOS \\
\hline FELIPE BORTOT CESAR & IGOR MATOS LAGO \\
\hline FELIPE CAMELO BIAGI & ITAMAR RIBEIRO DE OLIVEIRA \\
\hline FELIPE EDUARDO HATSUMURA & IVAN FERREIRA DE FREITAS \\
\hline FERNANDO BULLOS FILHO & JAIRON NASCIMENTO ALENCAR \\
\hline FERNANDO DE MARTINO & JAMIL ABDALLA SAAD \\
\hline FERNANDO MENDES SANT'ANNA & JAMIL RIBEIRO CADE \\
\hline FERNANDO STUCHI DEVITO & JOÃO ADDISON PESSOA \\
\hline FILIPE GOLDBERG & JOÃO ALEXANDRE FARJALLA CARACAS \\
\hline FLAVIO ARAUJO CANEDO & JOÃO ALFREDO FALCAO CUNHA LIMA \\
\hline FLAVIO BORGES & JOÃO BATISTA DE OLIVEIRA NETO \\
\hline FLAVIO JOSE ROCHA DE SOUZA & JOÃO BATISTA FREITAS GUIMARÃES \\
\hline FLAVIO PASSOS BARBOSA & JOÃO BATISTA LOPES LOURES \\
\hline FLAVIO ROBERTO AZEVEDO DE OLIVEIRA & JOÃO BOSCO DA SILVA FILHO \\
\hline FRANCISCO CLAUDIO COUTO FALCÃO & JOÃO CARLOS BELO LISBOA DIAS \\
\hline FRANCISCO JUAREZ CRUZ DE VASCONCELOS & JOÃO EDUARDO TINOCO DE PAULA \\
\hline FILHO & JOÃO FELIPE BARROS DE TOLEDO \\
\hline FREDERICK MALTA BUARQUE DE GUSMÃO & JOÃO FRANCISCO DE SOUZA \\
\hline FREDERICO AUGUSTO LIMA E SILVA & JOÃO GUILHERME ALVES LOURES \\
\hline GALO ALFREDO MALDONADO ANDRADE & JOÃO LOURENÇO VILLARI HERRMANN \\
\hline GEORGE CESAR XIMENES MEIRELLES & JOÃO LUCAS O'CONNELL \\
\hline GERALDO LUIZ DE FIGUEIREDO & JOÃO LUIZ DE ALENCAR ARARIPE FALCÃO \\
\hline GERSON MIRANDA & JOÃO MIGUEL MALTA DANTAS \\
\hline GIANCARLO GONÇALVES & JOÃO ORAVIO DE FREITAS JUNIOR \\
\hline GIANCARLO RABELO E SILVA & JOÃO PAULO ZOUVI \\
\hline GILBERTO GUILHERME AJJAR MARCHIORI & JOCELINO PEREGRINO SOARES \\
\hline GILBERTO HEINECK & JORGE DE CAMARGO NETO \\
\hline GILBERTO LAHORGUE NUNES & JORGE LUIS HADDAD \\
\hline GLAUCO SOARES MAIA PIASSI & JORGE PEREGRINO BRAGA \\
\hline GUILHERME ALVES & JOSÉ AIRTON DE ARRUDA \\
\hline GUILHERME BRANDÃO ALMEIDA & JOSÉ ALBINO MALTA DE SOUZA \\
\hline GUILHERME ESHER & JOSÉ ALFREDO SEJOPOLES \\
\hline GUILHERME FERRAGUTTI ATTIZZANI & JOSÉ ANTONIO JATENE \\
\hline GUILHERME RODRIGUES MAFFEIS & JOSÉ ANTONIO MARIN-NETO \\
\hline GUSTAVO A. BARRUEO & JOSÉ ARMANDO MANGIONE \\
\hline GUSTAVO ADOLFO BRAVO RANDO & JOSÉ ARY BOECHAT E SALLES \\
\hline
\end{tabular}




\section{APÊNDICE \\ Lista de Associados da SBHCI que se mantiveram efetivamente contribuindo com dados à CENIC durante o biênio 2006-2007}

\begin{tabular}{|c|c|}
\hline JOSÉ AUGUSTO MARCONDES DE SOUZA & LUIZ CARLOS TELES CORREA \\
\hline JOSÉ AUGUSTO ROCHA ARAUJO & LUIZ CLAUDIO MENDES CARVALHO \\
\hline JOSÉ CARLOS FELIPE ABUD & LUIZEDUARDO KOENIG SÃO THIAGO \\
\hline JOSÉ CARLOS RAIMUNDO BRITO & LUIZ EMILIO SALOME \\
\hline JOSÉ DEL CARMEN SOLANO ALIAGA & LUIZHEITOR DEMOLINARI JUNIOR \\
\hline JOSÉ ERIRTONIO FAÇANHA BARRETO & LUIZJUNYAKAJITA \\
\hline JOSÉ FABIO FABRIS JUNIOR & LUIZ KOHN \\
\hline JOSÉ GUILHERME CARNEIRO & MAEVE DE BARROS CORREIA \\
\hline JOSÉ LUIS ARREDONDO QUINONES & MANOEL AUGUSTO BAPTISTA ESTEVES \\
\hline JOSÉ LUIS ATTAB DOS SANTOS & MANUEL NICOLAS CANO \\
\hline JOSÉ MARCONI ALMEIDA SOUZA & MARCEL ROGERS RAVANELLI \\
\hline JOSÉ MARIA DIAS AZEREDO BASTOS & MARCELO BASTOS BRITO \\
\hline JOSÉ MARIANI JUNIOR & MARCELO EMILIO ARNDT \\
\hline JOSÉ MATOS BRITO CASTELLO BRANCO & MARCELO GOES ALVES DA SILVA \\
\hline JOSÉ RICARDO BUENO DE MORAES BIROLLI & MARCELO JOSÉ CANTARELLI \\
\hline JOSÉ ROBERTO MAIELLO & MARCELO LEMOS RIBEIRO \\
\hline JOSÉ RONALDO MONT ALVERNE FILHO & MARCELO SABEDOTTI \\
\hline JOSÉ WALTER MENDES NOGUEIRA & MARCIO ANDRADE DE OLIVEIRA \\
\hline JOSILAVIO DE ALMEIDA ARAUJO & MARCIO ANTONIO DOS SANTOS \\
\hline JULIO CESAR FRANCISCO VARDI & MARCIO AUGUSTO TRUFFA \\
\hline JULIO CESAR MACHADO ANDREA & MARCIO JOSÉ MONTENEGRO DA COSTA \\
\hline JULIO DE PAIVA MAIA & MARCO ANTONIO NAZARE CASTRO \\
\hline JUNIOR CAMILO DE QUEIROZ & MARCO ANTONIO PERIN \\
\hline KLEBER BOMFIM ARAUJO MARTINS & MARCO CESAR MIGUITA \\
\hline LA HORE CORREA RODRIGUES & MARCOS ANTONIO MARINO \\
\hline LAERCIO FONSECA ANTELO & MARCOS FLAVIO MOELLMAN RIBEIRO \\
\hline LAZARO CLAUDOVINO GARCIA & MARCOS FRANCHETTI \\
\hline LEANDRO ASSUNPÇÃO CORTES & MARCOS JULIANO DE ABREU \\
\hline LEANDRO COUMBIS MANDALOUFAS & MARCOS KYOSHI SUMITA \\
\hline LEANDRO DE CARVALHO PEREIRA & MARCUS NOGUEIRA DA GAMA \\
\hline LEANDRO LOBÃO LUZ FILHO & MARDEN ANDRE TEBET \\
\hline LEONARDO ALVES & MARIA CRISTINA MEIRA FERREIRA \\
\hline LEONARDO ALVES BATISTA & MARIA DA CONCEIÇÃO ALVES PINTO \\
\hline LEONARDO AVANY NUNES & MARIA SANALI MOURA DE OLIVEIRA PAIVA \\
\hline LEONARDO COGO BECK & MARIOI. M. BONILLA \\
\hline LEONARDO FURTADO OLIVEIRA & MATEUS ROSSATO \\
\hline LEONARDO JOSÉ DUARTE SILVA & MAURICIO LOPES PRUDENTE \\
\hline LEONARDO MARTINS BARROSO & MAURICIO REZENDE BARBOSA \\
\hline LEONIDAS ALVARENGA HENRIQUES & MAURO ISOLANI PENA \\
\hline LIVIA R. FERNANDES & MICHELI ZANOTI GALON \\
\hline LIVIA RIBEIRO FERNANDES & MIGUEL ANTONIO NEVES RATI \\
\hline LUCIANA CONSTANT DAHER & MILTON DE MACEDO SOARES NETO \\
\hline LUCIANO DE MOURA SANTOS & MILTON FERREIRA NEVES FILHO \\
\hline LUCIANO MAURICIO DE ABREU FILHO & MONICA BUCHALLA \\
\hline LUCIANO NOGUEIRA LIBERATO DE SOUSA & MOYSES DE OLIVEIRA LIMA FILHO \\
\hline LUDIMILA PEREIRA TARTUCE & MURILLO KENJI FURUKAWA \\
\hline LUDMILLA ALMEIDA ROCHA RIBEIRO DE OLIVEIRA & NAHALIEL PESSOA RODRIGUES \\
\hline LUIS ANTONIO OLIVEIRA FERREIRA & NILSON BORGES RAMOS \\
\hline LUIS GUSTAVO DE MIRANDA MARQUES & NILSON DE MOURA FE FILHO \\
\hline LUIS MARIA CABRERA YORDI & NILTON CARLOS SPINOLA MACHADO \\
\hline LUIZ ALBERTO MATTOS & NORBERTO TOAZZA DUDA \\
\hline LUIZ ANTONIO GUBOLINO & NORIAKITAKESHITA \\
\hline
\end{tabular}




\section{APÊNDICE}

Lista de Associados da SBHCI que se mantiveram efetivamente contribuindo com dados à CENIC durante o biênio 2006-2007

\begin{tabular}{|c|c|}
\hline OSCAR BITTENCOURT LINS NETO & RODRIGO VERNEY CASTELLO BRANCO \\
\hline OTAVIO EBOLI & ROGERIO DE BARROS WANDERLEY \\
\hline PABLO TOME TEIXEIRENSE & ROGERIO DE CASTRO PIMENTEL \\
\hline PAULO HENRIQUE JORGE & ROGERIO EDUARDO GOMES SARMENTO LEITE \\
\hline PAULO MAURICIO PIA DE ANDRADE & ROGERIO FELIPPE TIOSSI \\
\hline PAULO RENATO MERCIO MACHADO & RONE MARQUES PADILHA \\
\hline PAULO RICARDO AVANCINI CARAMORI & RONEI BOSCO DE MATOS \\
\hline PAULO RICARDO FRANCIOZI DE GOIS & ROSALY GONÇALVES \\
\hline PAULO ROBERTO FERREIRA TARTUCE FILHO & SALVADOR ANDRE BAVARESCO CRISTOVÃO \\
\hline PAULO ROGERIO DE OLIVEIRA MONTEIRO & SAMIR SEME ARAB REIS \\
\hline PAULO SERGIO DE OLIVEIRA & SANDRO ANTONIO TEIXEIRA \\
\hline $\begin{array}{l}\text { PEDRO ABILIO RIBEIRO RESECK } \\
\text { PFDRO AIVFS IFMOS NFTO }\end{array}$ & SANDRO OLIVEIRA SACRE \\
\hline $\begin{array}{l}\text { PEDRO ALVES LEMOS NETO } \\
\text { PEDRO AUGUSTO PASCOLI }\end{array}$ & SERGIO CORREA PRATA \\
\hline PEDRO BERALDO DE ANDRADE & SERGIO GUSTAVO TARBINE \\
\hline PEDRO EDUARDO HORTA & SERGIO KREIMER \\
\hline PEDRO ESBERARD ARAGAO BELTRÃO & SERGIO LUIS BERTI \\
\hline PEDRO GOMES DE ALMEIDA GARZON & SIDNEY MUNHOZJUNIOR \\
\hline PEDRO PAULO NEVES DE CASTRO & SILVIA MACHADO ABREU \\
\hline RAIMUNDO ANTONIO DE MELO & SILVIO GIOPATTO \\
\hline RALDIR BASTOS FILHO & SILVIO SERGIO PONTES CAMARA \\
\hline REGIS ARY MOSSMANN & STEFAN COSTA DA SILVEIRA \\
\hline RENATO GIESTAS SERPA & TAMMUZ FATTAH \\
\hline RICARDO CESAR CAVALCANTI & TARCIO FIGUEIREDO SILVA \\
\hline RICARDO JOSÉ ARAUJO SILVEIRA & TARCISIO MARIZ MAIA FILHO \\
\hline RICARDO JOSÉ TOFANO & THIAGO NOBREGA DE OLIVEIRA \\
\hline RICARDO LASEVITCH & TIAGO PORTO DI NUCCI \\
\hline RICARDO MONTEIRO LOURENÇO & TRAJANO ALFONSO \\
\hline RICARDO PERESSONI FARACO & UBIRAJARA LIMA FILHO \\
\hline RICARDO UEDA & ULISES ENRIQUE ACUNA SOLORZANO \\
\hline RICARDO ZAUITH SILVA & VAGNER VINICIUS FERREIRA \\
\hline RIZZIERI MOURA GOMES & VALDEMAR DE SOUZA OLIVEIRA JUNIOR \\
\hline ROBERTO DE ALMEIDA CESAR & VALERIO FUKS \\
\hline ROBERTO JOSÉ DA PAIXÃO & VALTER CORREIA DE LIMA \\
\hline ROBERTO JOSÉ DE QUEIROZ CREPALDI & VICTOR DUTRA VIEIRA FILHO \\
\hline $\begin{array}{l}\text { ROBERTO LEO DA SILVA } \\
\text { ROBERTO IUCIO DF GUSMÃO VERCOZA }\end{array}$ & VINICIUS DAHER VAZ \\
\hline $\begin{array}{l}\text { ROBERTO OTSUBO } \\
\text { ROSMAO VERCOA }\end{array}$ & VINICIUS FRAGA MAURO \\
\hline ROBERTO REIS VIEIRA & VIRGILIO RIBEIRO FRANCO JUNIOR \\
\hline ROBERTO VIEIRA BOTELHO & VITOR GOMES BARRETO \\
\hline ROBSON BUENO DE CARVALHO & VITOR OSORIO GOMES \\
\hline RODOLFO ALBERTO SILVEIRA MALTA ALENCAR & WALASSE ROCHA VIEIRA \\
\hline RODOLFO DE FRANCO CARDOSO & WALKIMAR URURAY GLORIA VELOSO \\
\hline RODOLFO STAICO & WALTER BENEDUZZI FIOROTTO \\
\hline RODRIGO COSTA QUINTÃO & WELLINGTON BORGES CUSTODIO \\
\hline RODRIGO DE FRANCO CARDOSO & WENCESLAU DE CARVALHO RIBAS \\
\hline RODRIGOJULIO CERCI & WESLEY FERRAZ DA SILVEIRA \\
\hline RODRIGO TRAJANO SANDOVAL PEIXOTO & WILSON ALBINO PIMENTEL FILHO \\
\hline
\end{tabular}

